\title{
Energieautark arbeitendes Indoor-Smart-Gardening-System mit drahtlosem Monitoring und automatisierter Bewässerung
}

\author{
Elke Mackensen, Axel Rombach, Aaron Spitznagel, Julius Klose \\ Hochschule Offenburg; Fakultät Elektrotechnik, Medizintechnik und Informatik; \\ Badstraße 24; 77652 Offenburg
}

\section{Zusammenfassung}

Smart Home-/Smart-Building-Anwendungen sind ein stetig wachsender Markt. Smart Gardening ist ein Beispiel dafür, Nutzern mehr Komfort und eine bessere Lebensqualität zu Hause oder in Bürogebäuden zu ermöglichen. Im Rahmen dieses Beitrags wird die Entwicklung eines Indoor-SmartGardening-Systems mit dem Fokus auf energieautarkes Arbeiten vorgestellt. Herzstück des Systems ist ein 3D-gedruckter Blumentopf für einzelne Pflanzen mit integrierter Elektronik zum Monitoring der wichtigsten Pflanzenparameter und einem integrierten Wasserreservoir mit Tauchpumpe für das automatisierte Bewässern der Pflanze. Energy Harvesting per Solarzellen ermöglicht ein energieautarkes Arbeiten des Blumentopfes. Eine selbstentwickelte Low-Power-Funkschnittstelle im Blumentopf und ein externes Gateway ermöglichen die drahtlose Vernetzung mehrerer Pflanzen. Das Gateway dient zur Auswertung der Pflanzenparameter, der Ansteuerung der im Netzwerk vorhandenen Blumentöpfe und als Benutzerinterface.

Keywords: Smart Home, Smart Gardening, Wireless Sensor Nodes, Energy Harvesting, Low-CostFüllstandssensor

\section{Einführung und Stand der Technik}

Auf Grund verfügbarer Funktechniken und Fortschritte in der Informationstechnik sind Smart-Home-/Smart-Building-Applikationen ein stark wachsender Markt [1]. Smart Gardening ist ein typisches Beispiel einer Smart-Home-/ Smart-Building-Anwendung. Im Detail bedeutet Smart Gardening per Messung diverse Parameter der Pflanze (z.B. Bodenfeuchte, Licht- und Temperatur-verhältnisse, Luftfeuchtigkeit, Nährstoffgehalt usw.) zu erfassen, auszuwerten und entsprechende Aktionen auf Grund der Messparameter einzuleiten. Dazu gehören z.B. eine automatisierte Bewässerung der Pflanze und/oder den Benutzer darüber zu informieren, welche Aktionen für eine einzelne Pflanze aus einem Pool von $n$ Pflanzen notwendig sind.

Smart-Gardening-Systeme stehen im Fokus der Forschung, erste Systeme sind am Markt verfügbar [2]-[9]. Man unterscheidet zwischen Indoor- und Outdoor-Gardening-Systemen, die aus mehreren vernetzten Sensorkonten bestehen, welche die unterschiedlichen Pflanzenparameter messen. In vielen Fällen insbesondere im Outdoor-Bereich - werden die gemessenen Parameter per Funk zu einem
Gateway oder direkt in eine Internet-Cloud übertragen. Software-Anwendungen auf dem Gateway ermöglichen es, die Daten dem Nutzer auf entsprechenden Endgeräten wie PC, Smartphone oder Tablets verfügbar zu machen. Indoor-Smart-Gardening-Systeme unterscheiden sich zumeist in den folgenden Merkmalen (siehe Tab. 1): Art der Pflanzenparameter, die gemessen werden, Grad der Automatisierung (z.B. automatische Bewässerung und Düngung), Art der Energieversorgung und Art der drahtlosen Kommunikationstechnik, welche einen Einfluss auf die Kommunikationsreichweite in einem Netzwerk von $n$ Pflanzen und den Energiebedarf der Sensorknoten hat

In der Literatur beschriebene Indoor-SmartGardening-Systeme haben oftmals die Nachteile eines externen Wassertanks zur Bewässerung der Pflanze, zudem besteht die Energieversorgung des Systems zumeist aus Primärbatterien, die nach einer bestimmten Zeit ausgewechselt werden müssen. Außerdem wird eine Vernetzung eines Pools aus $n$ Planzen nur bedingt unterstützt. 
Tab. 1: Vergleich verschiedener Indoor-Smart-Gardening-Systeme

\begin{tabular}{|c|c|c|c|c|}
\hline & $\begin{array}{c}\text { System } 1 \text { [6]; } \\
\text { Forschung }\end{array}$ & $\begin{array}{c}\text { System } 2 \text { [7]; } \\
\text { Forschung }\end{array}$ & $\begin{array}{l}\text { System } 3 \text { [8]; } \\
\text { Kommerziell }\end{array}$ & $\begin{array}{c}\text { System } 4 \text { [9]; } \\
\text { Kommerziell, } \\
\text { aber nicht mehr } \\
\text { verfügbar }\end{array}$ \\
\hline $\begin{array}{l}\text { Gemessene } \\
\text { Pflanzen- } \\
\text { parameter }\end{array}$ & $\begin{array}{l}\text { Bodenfeuchte, } \\
\text { Temperatur, } \\
\text { Luftfeuchtigkeit, }\end{array}$ & $\begin{array}{l}\text { Bodenfeuchte, } \\
\text { Licht }\end{array}$ & Bodenfeuchte & $\begin{array}{l}\text { Bodenfeuchte, } \\
\text { Temperatur, Licht, } \\
\text { Nährstoffgehalt }\end{array}$ \\
\hline $\begin{array}{l}\text { Ansteuerung } \\
\text { von Aktuatoren }\end{array}$ & $\begin{array}{l}\text { Automatische } \\
\text { Pflanzen- } \\
\text { bewässerung }\end{array}$ & $\begin{array}{l}\text { Automatische } \\
\text { Pflanzen- } \\
\text { bewässerung }\end{array}$ & $\begin{array}{l}\text { Automatische } \\
\text { Pflanzen- } \\
\text { bewässerung }\end{array}$ & $\begin{array}{l}\text { Automatische } \\
\text { Pflanzen- } \\
\text { bewässerung und } \\
\text { Düngung }\end{array}$ \\
\hline Kommentar & $\begin{array}{l}\text { Sensoren/Aktoren } \\
\text { sind direkt mit } \\
\text { einem Arduino } \\
\text { Board verbunden }\end{array}$ & $\begin{array}{l}\text { Externes Wasser- } \\
\text { reservoir, } \\
\text { Sensoren/Aktoren } \\
\text { sind direkt mit } \\
\text { einem Raspberry- } \\
\text { Pi-Board } \\
\text { verbunden }\end{array}$ & $\begin{array}{l}\text { Externes Wasser- } \\
\text { reservoir }\end{array}$ & $\begin{array}{l}\text { Die gesamte } \\
\text { Elektronik ist in } \\
\text { einen Blumentopf } \\
\text { integrierte }\end{array}$ \\
\hline $\begin{array}{l}\text { Energie- } \\
\text { versorgung } \\
\text { und } \\
\text { energetische } \\
\text { Lebensdauer }\end{array}$ & $\begin{array}{l}\text { Keine } \\
\text { Informationen } \\
\text { verfügbar }\end{array}$ & $\begin{array}{l}12 \text { VDC; Keine } \\
\text { Informationen zur } \\
\text { energetischen } \\
\text { Lebensdauer }\end{array}$ & $\begin{array}{l}2 \text { AAA Batterien; } \\
\text { Keine } \\
\text { Informationen zur } \\
\text { energetischen } \\
\text { Lebensdauer }\end{array}$ & $\begin{array}{l}4 \text { AA Batterien; } \\
\text { energetische } \\
\text { Lebensdauer } \\
6 \text { Monate }\end{array}$ \\
\hline $\begin{array}{l}\text { Drahtlose } \\
\text { Kommuni- } \\
\text { kationstechnik } \\
\text { von den } \\
\text { Sensoren/ } \\
\text { Aktoren zu } \\
\text { einem Gateway } \\
\text { und Netzwerk } \\
\text { Funktionalität }\end{array}$ & $\begin{array}{l}\text { Kein Gateway; } \\
\text { Arduino-Boards } \\
\text { sind zu einer } \\
\text { Thingspeak Cloud } \\
\text { via Ethernet oder } \\
\text { Wi-Fi konnektiert, } \\
\text { auf die Benutzer } \\
\text { per Spartphoe- } \\
\text { App zugreifen } \\
\text { können } \\
\end{array}$ & $\begin{array}{l}\text { Kein Gateway; } \\
\text { Keine Netzwerk- } \\
\text { funktionalität. } \\
\text { Benutzer } \\
\text { bekommen } \\
\text { Informationen per } \\
\text { email }\end{array}$ & $\begin{array}{l}\text { Keine Netzwerk- } \\
\text { funktionalität. } \\
\text { Eine LED } \\
\text { informiert über zu } \\
\text { wenig Wasser im } \\
\text { Wasserreservoir }\end{array}$ & $\begin{array}{lr}\text { Bluetooth } & \text { V4.0 } \\
\text { BLE; } & \text { Kein } \\
\text { Gateway; } & \text { Die } \\
\text { Elektronik } & \text { des } \\
\text { Blumentopfes } & \\
\text { kann per } & \text { Smart } \\
\text { Phone } & \text { App } \\
\text { konnektiert } & \\
\text { werden } & \end{array}$ \\
\hline
\end{tabular}

In Rahmen von diesem Beitrag wird die Entwicklung eines innovativen Indoor-SmartGardening-Systems vorgestellt, dessen Fokus auf energieautarkem Arbeiten, einer einfachen Bedienbarkeit für den Benutzer und die optimale Pflege individueller Pflanzen liegt.

Im Folgenden wird zunächst auf die Architektur und die Funktionalität des Systems eingegangen, danach werden Messergebnisse des Indoor-Smart-Gardening-Systems präsentiert. Ein Schwerpunkt des Beitrags ist insbesondere die Betrachtung des energieautarken Arbeitens des Systems, das durch Energy-Harvesting und eine selbstentwickelte Low-Power-Funkschnittstelle erreicht wird.

\section{Architektur und Funktionalität des Indoor- Smart-Gardening-Systems}

Das entwickelte System besteht aus drei Hauptbestandteilen (siehe Abb. 1): Wichtigster Bestandteil ist das Netzwerk aus 3Dgedruckten intelligenten Blumentöpfen für individuelle Pflanzen. In dem Blumentopf ist eine Low-Power-Funkschnittstelle integriert. Ein Raspberry-Pi3-System dient als Gateway für das Netzwerk von $n$ Blumentöpfen. Das Gateway dient zur Auswertung der Pflanzenparameter, der Ansteuerung der Blumentöpfe im Netzwerk und als Benutzerschnittstelle für Software-APPs auf Smartphone, PC oder ähnlichem.

Der intelligente Blumentopf (siehe Abb. 2) besteht aus einem integrierten Wasserreservoir mit einer Tauchpume für die automatische Bewässerung der Pflanze und einer integrierten Elektronik zum Monitoring verschiedenen Pflanzenparametern und des Wasserstandes im Wasserreservoir. EnergyHarvesting per Solarzellen ermöglichen ein energieautarkes Arbeiten des Blumentopfes.

Eine detaillierte Architektur der integrierten Elektronik des intelligenten Blumentopfes ist in Abb. 3 dargestellt. Die geerntete Energie wird in einem Akku mit einer Kapazität von 
350 mAh gespeichert. Die Elektronik besteht aus diversen Sensoren. Ein Multisensorchip ermöglicht die Erfassung der Umgebungsbedingungen der Pflanzen. Der zum Einsatz kommende Multisensorchip ermöglicht die Messung der Umgebungstemperatur, der Luftfeuchtigkeit und des Luftdrucks. Ein weiterer Sensor ermöglicht die Messung der Bodenfeuchte, so dass sichergestellt werden kann, dass die Pflanze optimal mit Wasser versorgt wird. Zudem kommt ein selbstentwickelter Sensor zum Einsatz, der den Wasserstand im Wasserreservoir überprüft.

Ein Mikrocontroller und ein PowerManagement sind weitere Bestandteile der integrierten Elektronik. Das PowerManagement ermöglicht energieautarkes Arbeiten des Blumentopfes. Verschiedene Methoden werden angewendet, um den Energieverbrauch der Elektronik zu minimieren:

- Ausnutzung der Low-Power-Modes der einzelnen Komponenten.

- Einsatz von Komponenten mit niedrigen Leckströmen.

- Verwendung einer selbstentwickelten energieoptimierten Funkschnittstelle für die drahtlose Kommunikation mit dem Gateway, die bei $868 \mathrm{MHz}$ arbeitet.

- Implementierung einer Ein-/Ausschaltlogik für die einzelnen Komponenten. Einzige wirklich dauerhaft betriebene Komponente des Systems ist eine Real-Time-Clock.

Der eingesetzte Mikrocontroller verfügt über einen FRAM-Speicher für die Speicherung der Sensordaten und der Firmware. Der Mikrocontroller erfasst alle Sensordaten, wertet diese aus, initiiert eine Bewässrung und überträgt relevante Daten zu dem RaspberryPI3-basiernden Gateway. Der Raspberry-PI3 wurde auf Grund seiner vielen standardisierten Schnittstellen ausgewählt. Das Gateway wird mit einer konstanten 5-V-DC-Spannung versorgt. Das Gateway wurde durch die gleiche 868-MHz-Funkschnittstelle erweitertet, welche auch in der integrierten Elektronik des Blumentopfes enthalten ist. Das Gateway empfängt von allen vorhandenen Blumentöpfen die übermittelten Daten und macht sie für weitere Softwareanwendungen verfügbar. Ein lokaler Node-Red-Server ermöglicht den Zugriff auf die Daten per WiFi oder Ethernet.

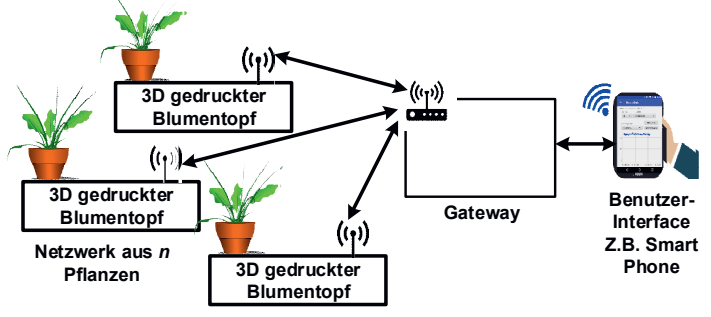

Abb. 1: Vereinfachte Architektur des IndoorSmart-Gardening-Systems

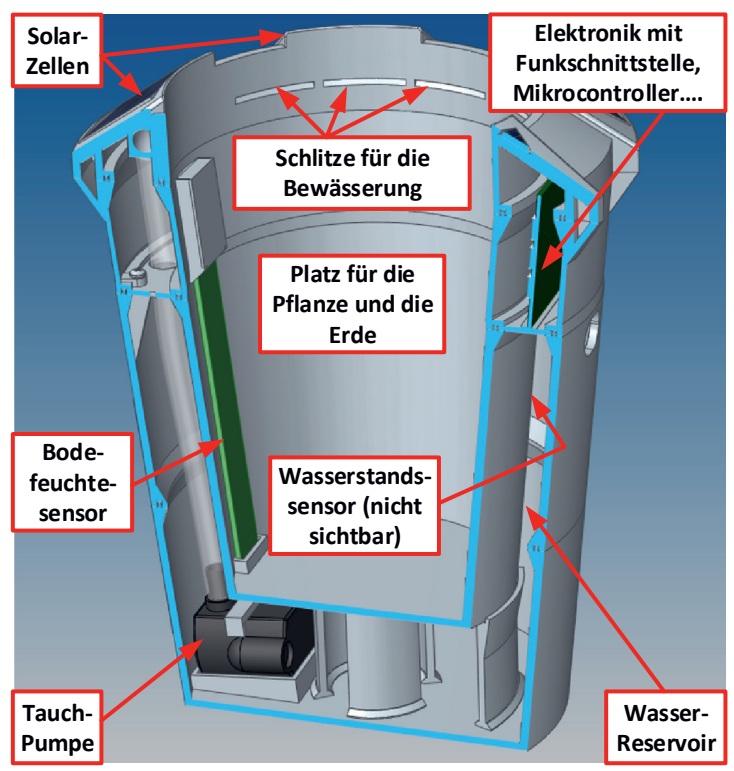

Abb. 2: Querschnitt durch den 3Dgedruckten intelligenten Blumentopf

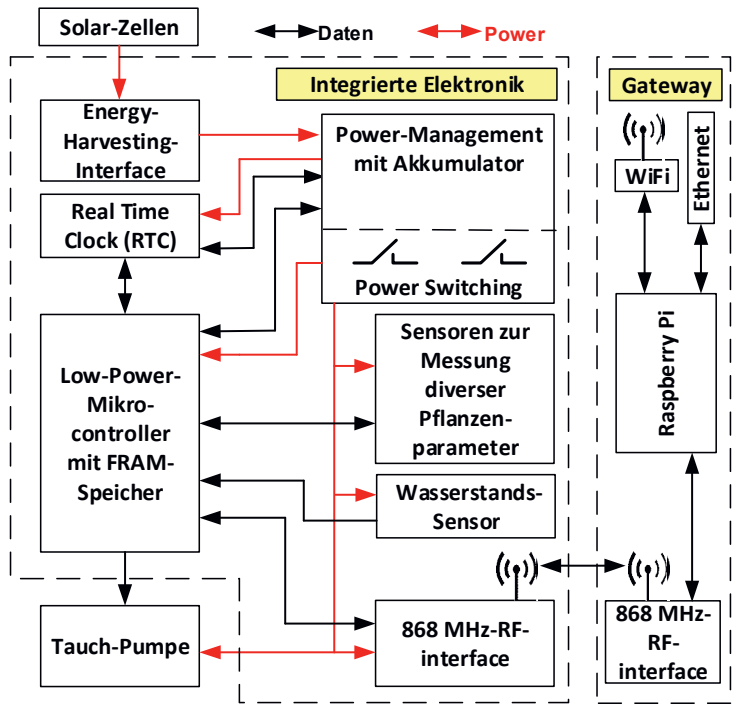

Abb. 3: Detaillierte Architektur des IndoorSmart-Gardening-Systems 


\section{Messdatenerfassung des Blumentopfes}

Wie bereits beschrieben, lassen sich mit dem eingesetzten Multisensorchip Temperatur, Luftdruck und Luftfeuchtigkeit erfassen. Abb. 4 zeigt als Beispiel die Messung der Temperatur in einem Innenraum in der Nähe eines Fensters am 28. August 2018.

Die Messung der Bodenfeuchte basiert auf einem kapazitiven Sensor. Die sich auf Grund der Bodenfeuchte ändernde Kapazität ist in einen Oszillatorschaltkreis eingebunden. Dessen sich ändernde Frequenz wird mit dem Mikrocontroller gemessen. Um genaue Messungen zu erhalten, muss der Sensor in Abhängigkeit der Erde bzw. des Pflanzensubstrates, in welchem die Pflanze eingebettet ist, kalibriert werden. Abb. 5 zeigt die Messung der Bodenfeuchte mit dem Smart-Gardening-System. Zu erkennen sind zwei Bewässerungs-Vorgänge. Die eher langsamen Messwertänderungen resultieren aus der langsamen Ausbreitung des Wassers in der Erde.

Zur Messung des Wasserstandes in dem Wasserreservoir des Blumentopfes kommt ein selbstentwickelter Sensor zum Einsatz, mit dem sich sechs unterschiedliche Wasserhöhen messen lassen (siehe Abb. 6). Der Sensor besteht aus einem resistiven Spannungsteiler. Der Spannungsteiler besteht aus Goldkontakten, die durch den elektrischen Widerstandes des Wassers geschlossen werden. D.h. der komplette Widerstand des Spannungsteilers ändert sich auf Grund der Höhe des Wasserstandes im Wasserreservoir. Eine nachfolgende Schaltung verstärkt den Spannungsabfall an dem resistiven Spannungsteiler. Nach einer Digitalisierung der analogen Spannung erfolgt eine Auswertung der Messwerte mit dem Mikrocontroller. Abb. 7 zeigt die verstärkte Ausgangsspannung des Sensors in Abhängigkeit des Wasserstandes.

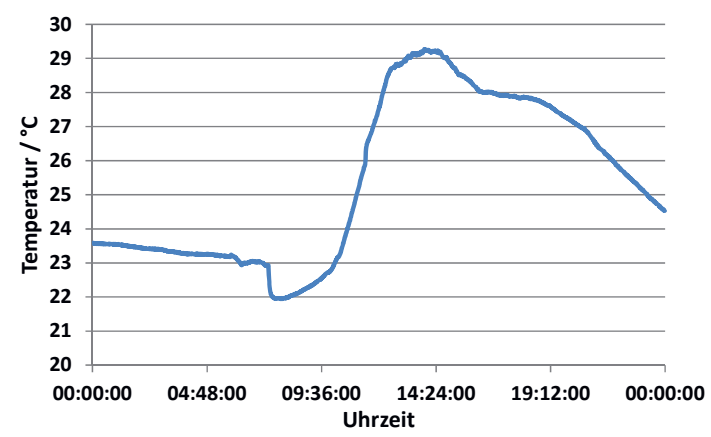

Abb. 4: Gemessene Umgebungstemperatur mit dem Smart-Gardening-System

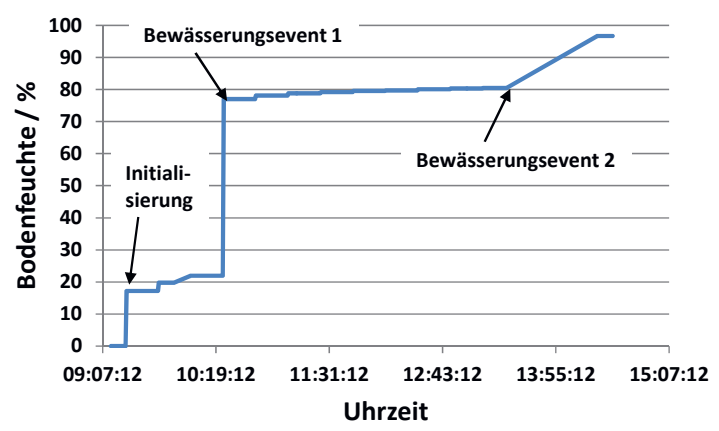

Abb. 5: Gemessene Bodenfeuchte mit dem Smart-Gardening-System

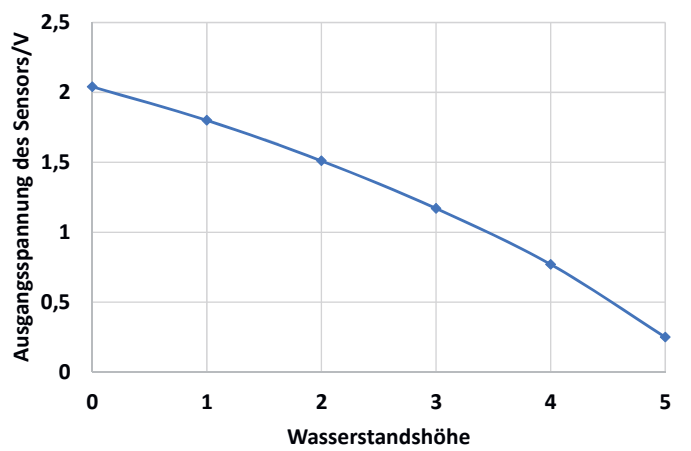

Abb. 7: Gemessene Ausgangsspannung des Wasserstandssensors in Abhängigkeit der Wasserstandshöhen im Wasserreservoir

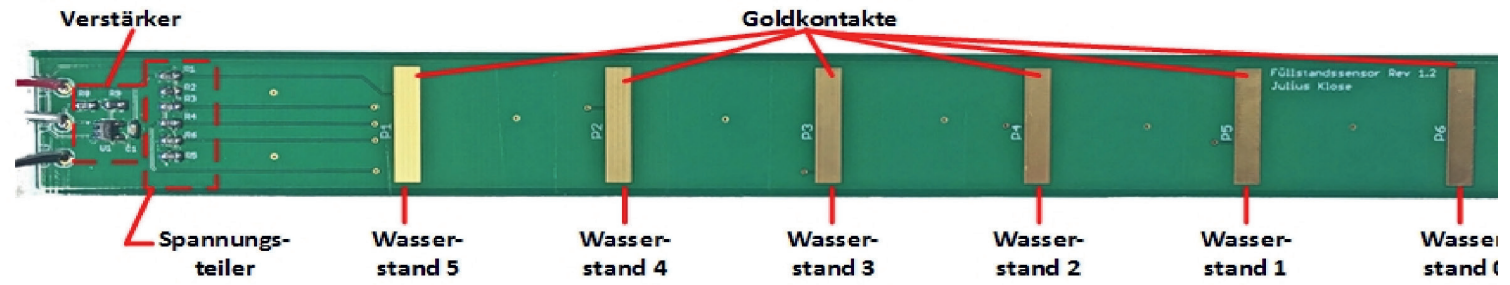

Abb. 6: Wasserstandssensor mit Goldkontakten 


\section{Drahtlose Kommunikation zwischen Blumentopfes und Gateway}

Die Entwicklung energieautarker, drahtlos kommunizierender Smart-Home-Anwendung ist nur möglich bei der Realisierung einer entsprechenden Low-Power-Funkschnittstelle. Das Smart-Gardening-System nutz für die drahtlose Kommunikation einen 868-MHzTransceiver von ST Microelectronics. Dieser Transceiver wurde ausführlich evaluiert, um die Funkschnittstelle so zu konfigurieren, dass dieser möglichst wenig Energie benötigt [10][11]. Im Vergleich zu anderen Kommunikationstechnologien [12]-[13], hat die drahtlose Schnittstelle des Blumentopfes einen recht niedrigen Energieverbrauch [10]. Die Kommunikation des Gateways und der Blumentöpfe ist als Stern-Topologie implementiert. Die einzelnen Blumentöpfe haben einen zugewiesenen Zeit-Slot für die Kommunikation, damit lässt sich die Kommunikation am energiesparendsten realisieren. Insgesamt lassen sich an einem Gateway bis zu 20 Blumentöpfe betreiben, prinzipiell könnte man das Netzwerk auch noch auf mehr als 20 Blumentöpfe erweitern.

Die erreichbare Kommunikationsreichweite zwischen den Blumentöpfen und dem Gatway ist eine wichtige Größe für das Indoor-SmarGardening-System. Die mögliche Kommunikationsreichweite wurde in einem realistischen Einfamilienhaus gemessen (siehe Abb. 8). Für die Messung wurde das Gateway recht zentral im Haus platziert, die Blumentöpfe wurden auf andere Räume verteilt. An jedem Blumentopf wird die Sendeleistung soweit reduziert, bis das Gateway die Daten des Blumentopfes nicht mehr korrekt empfangen konnte.

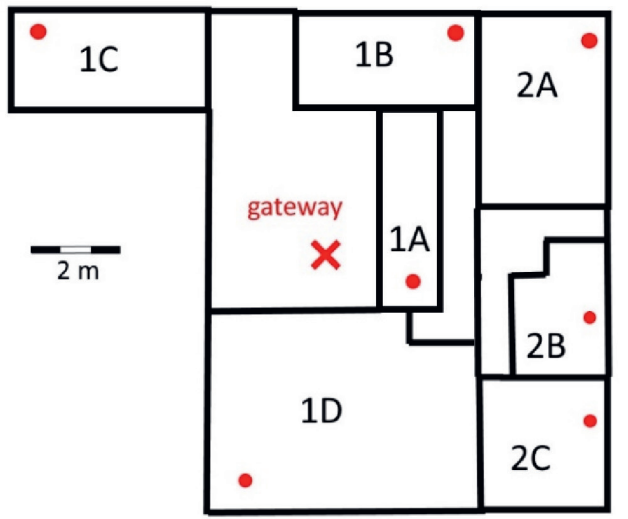

Abb. 8: Szenario in einem Haus, um die möglichen Kommunikationsabstände zwischen Gateway (rotes Kreuz) und den Blumentöpfen (rote Punkte) zu evaluieren
Die Ergebnisse der Messung sind in Tab. 2 dargestellt. Wie erwartet, haben die Anzahl der Wände und sonstigen Hindernisse natürlich einen Einfluss auf die fehlerfreie Übertragung. Die Ergebnisse zeigen jedoch, dass in einem Haus mit vielen Räumen und Türen, die Sendeleistung der drahtlosen Schnittstelle zumeist nicht mehr als $0 \mathrm{dBm}$ betragen muss. Diese geringe Sendeleistung ermöglicht enorme Energieeinsparungen bei gleichzeitig zuverlässiger Kommunikation.

Tab. 2: Gemessene minimale notwendige Sendeleistung für das Messszenario aus Abb. 8

\begin{tabular}{|l|l|l|l|}
\hline $\begin{array}{l}\text { Blumen- } \\
\text { topf } \\
\text { Nr. }\end{array}$ & $\begin{array}{l}\text { Direkte } \\
\text { Reich- } \\
\text { weite } \\
\text { zwischen } \\
\text { Blumen- } \\
\text { topf und } \\
\text { Gateway }\end{array}$ & $\begin{array}{l}\text { Anzahl } \\
\text { Wände } \\
\text { zwischen } \\
\text { Blumen- } \\
\text { topf und } \\
\text { Gateway }\end{array}$ & $\begin{array}{l}\text { Minimale } \\
\text { Sende- } \\
\text { leistung } \\
\text { für eine } \\
\text { noch } \\
\text { fehler- } \\
\text { freie } \\
\text { Kommu- } \\
\text { nikation }\end{array}$ \\
\hline 1A & $2,0 \mathrm{~m}$ & 1 & $-30 \mathrm{dBm}$ \\
\hline 1B & $5,7 \mathrm{~m}$ & 2 & $-22 \mathrm{dBm}$ \\
\hline 1C & $8,0 \mathrm{~m}$ & 2 & $-8 \mathrm{dBm}$ \\
\hline 1D & $5,3 \mathrm{~m}$ & 1 & $-20 \mathrm{dBm}$ \\
\hline 2A & $7,6 \mathrm{~m}$ & 3 & $-16 \mathrm{dBm}$ \\
\hline 2B & $6,0 \mathrm{~m}$ & 4 & $-3 \mathrm{dBm}$ \\
\hline 2C & $7,0 \mathrm{~m}$ & 5 & $5 \mathrm{dBm}$ \\
\hline
\end{tabular}

\section{Energetische Betrachtung des Gesamtsystems}

Das energetische Verhalten des intelligenten Blumentopfes wurde für ein bestimmtes Anwendungsszenario untersucht. Die Randbedingungen des Anwendungsszenarios sind wie folgt: Alle 10 Minuten führt das System mit den verschiedenen Sensoren Messungen aus und sendet die Messdaten an das Gateway. Die Sendeleitung des Transceivers beträgt $11 \mathrm{dBm}, 62$ Byte Payload-Daten werden an das Gateway gesendet, die Tauchpumpe läuft maximal 30 Sekunden pro Tag. Tab. 3 stellt die benötigte Energie der integrierten Elektronik inklusive Tauchpumpe pro Tag für das beschriebene Anwendungs-szenario dar. Die Ergebnisse basieren auf ausführlichen Messungen aus [11]. Es ist zu erkennen, dass durch das Ein-/Ausschalten der Komponenten versucht wird, möglichst große Standby-Zeiten der einzelnen Komponenten zu erreichen. Das System benötigt pro Tag in der Summe 8,28 J. Der verwendete Akkumulator hat eine Kapazität von $350 \mathrm{mAh}$ bei einer Spannung von 3,7 V. Unter der Annahme, dass $50 \%$ seiner Kapazität genutzt werden können, d.h. $175 \mathrm{mAh}$ oder $2331 \mathrm{~J}$, beträgt die energetische Lebensdauer des Systems ca. 280 Tage. 
Tab. 3: Benötigte Energie des intelligenten Blumentopfes

\begin{tabular}{|l|l|l|l|l|}
\hline \multicolumn{1}{|c|}{$\begin{array}{c}\text { System- } \\
\text { Komponente }\end{array}$} & $\begin{array}{c}\text { Aktive } \\
\text { Arbeitszeit } \\
\text { pro Tag/s }\end{array}$ & $\begin{array}{c}\text { Benötigte } \\
\text { Energie für die } \\
\text { Aktive } \\
\text { Arbeitszeit/J }\end{array}$ & $\begin{array}{c}\text { Standby-Zeit } \\
\text { pro Tag/s }\end{array}$ & $\begin{array}{c}\text { Benötigte } \\
\text { Energie für } \\
\text { die Standby- } \\
\text { Zeit/J }\end{array}$ \\
\hline $\begin{array}{l}\text { Block Power- } \\
\text { Management }\end{array}$ & 86400 & 6,5707200 & 0 & 0 \\
\hline Tauchpumpe & 30 & 0,015069000 & 86370 & 0 \\
\hline Real Time Clock & 5,76 & 0,000384912 & 86394,24 & 0,125444436 \\
\hline $\begin{array}{l}\text { Mikrokontroller und } \\
\text { Umgebungssensor }\end{array}$ & 14,4 & 0,039024000 & -- & -- \\
\hline $\begin{array}{l}\text { Mikrokontroller und } \\
\text { Bodenfeuchtesensor }\end{array}$ & 14,4 & 0,085536000 & -- & -- \\
\hline $\begin{array}{l}\text { Mikrokontroller und } \\
\text { Wasserstandssensor }\end{array}$ & 14,4 & 0,000950400 & -- & -- \\
\hline $\begin{array}{l}\text { Mikrokontroller und } \\
\text { Funkschnittstelle im } \\
\text { Sendemodus }\end{array}$ & 72 & 0,599040000 & -- & -- \\
\hline $\begin{array}{l}\text { Mikrokontroller und } \\
\text { Funkschnittstelle im } \\
\text { Empfangsmodus }\end{array}$ & 72 & 0,599040000 & -- & - \\
\hline Mikrocontroller inaktiv & -- & -- & 8684,8 & 0,000003960 \\
\hline $\begin{array}{l}\text { Funkschnittstelle } \\
\text { inaktiv }\end{array}$ & -- & -- & 86256 & 0,241948080 \\
\hline Summe benötigter Energie pro Tag/J & 7,909764312 & & 0,367396476 \\
\hline
\end{tabular}

Um zu überprüfen, ob die implementierten Solarzellen ausreichend Energie pro Tag liefern können, wurde die Ausgangsleistung der Solarzellen gemessen. Für die Messung wurde der Blumentopf an ein Fenster mit eher schlechten Lichtverhältnissen platziert. Die Messergebnisse sind in Abb. 9 dargestellt. Während der Messung von drei Stunden konnte eine Energie von insgesamt 547,2 J geerntet werden. Somit sind die Solarzellen ausreichend, um den Blumentopf energieautark zu betreiben.

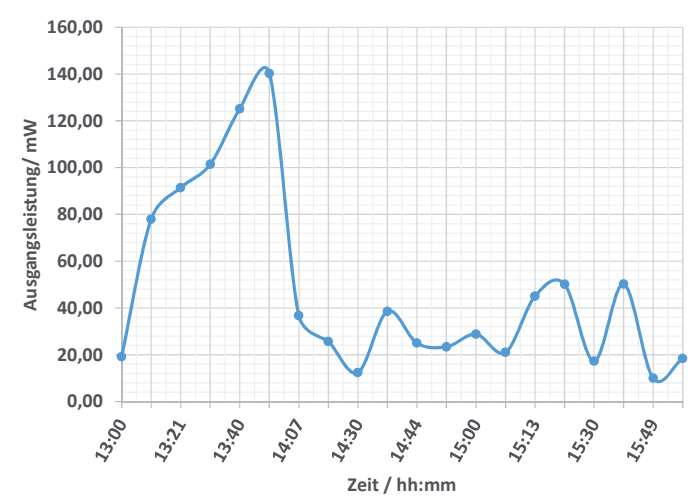

Abb. 9: Gemessene Ausgangsleistung der Solarzellen während drei Stunden

\section{Zusammenfassung}

Im Rahmen dieses Beitrags wurde die Entwicklung eines Indoor-Smart-GardeningSystems mit dem Fokus auf energieautarkes Arbeiten vorgestellt. Herzstück des Systems ist ein 3D-gedruckter Blumentopf für einzelne Pflanzen mit integrierter Elektronik zum Monitoring der wichtigsten Pflanzenparameter und einem integrierten Wasserreservoir mit Tauchpumpe für das automatisierte Bewässern der Pflanze. Energy Harvesting per Solarzellen ermöglicht ein energieautarkes Arbeiten des Blumentopfes. Die wichtigsten Eigenschaften des Smart-Gardening-System sind in Tab. 4 aufgeführt. Gegenüber existierenden und in der Literatur beschriebenen Ansätzen für Smart-GardeningAnwendungen, hat das System mehrere Vorteile:

- Das System lässt sich sowohl für Indoorals auch Outdoor-Anwendungen einsetzen.

- Die integrierte Elektronik könnte auf Grund des energieeffizienten Hardwaredesigns für eine große Anzahl anderer Anwendungen im Smart-Home-Bereich eingesetzt werden.

- Der größte Vorteil des präsentierten Systems ist, dass sogar unter Worst-Casebedingungen $(+11 \mathrm{dBm}$ Sendeleistung und wenig vorteilhaften Lichtverhältnissen), eine energetische Lebensdauer von mehreren Jahren erreicht werden kann, ohne Batterien zu ersetzen. 
Tab. 4: Eigenschaften des in diesem Beitrag präsentierten Indoor-SmartGardening-Systems

\begin{tabular}{|c|c|}
\hline Eigenschaften & Beschreibung \\
\hline $\begin{array}{l}\text { Gemessene } \\
\text { Pflanzen- } \\
\text { parameter }\end{array}$ & $\begin{array}{l}\text { Bodenfeuchte, } \\
\text { Umgebungstemperatur, } \\
\text { Luftfeuchtigkeit, } \\
\text { Luftdruck }\end{array}$ \\
\hline $\begin{array}{l}\text { Ansteuerung von } \\
\text { Aktuatoren }\end{array}$ & $\begin{array}{l}\text { Automatisierte } \\
\text { Bewässerung }\end{array}$ \\
\hline Kommentar & $\begin{array}{l}\text { Die gesamte Elektronik } \\
\text { ist in einen Blumentopf } \\
\text { integriert }\end{array}$ \\
\hline $\begin{array}{l}\text { Energie- } \\
\text { versorgung und } \\
\text { energetische } \\
\text { Lebensdauer }\end{array}$ & $\begin{array}{l}\text { Integrierte Elektronik des } \\
\text { Blumentopfes: Energy } \\
\text { Harvesting mit Solar- } \\
\text { zellen. Energetische } \\
\text { Lebensdauer unendlich; } \\
\text { Gateway (ein Raspberry } \\
\text { Pi3): 5-V-DC-Netzteil }\end{array}$ \\
\hline $\begin{array}{l}\text { Drahtlose } \\
\text { Kommuni- } \\
\text { kationstechnik } \\
\text { von den } \\
\text { Sensoren/ } \\
\text { Aktoren zu einem } \\
\text { Gateway } \\
\text { und Netzwerk } \\
\text { Funktionalität }\end{array}$ & $\begin{array}{l}\text { 868-MHz-Funkschnitt- } \\
\text { stelle: Line-of-Sight- } \\
\text { Kommunikationsreich- } \\
\text { weite: } 80 \text { m @ } 5 \text { dBm; } \\
\text { Bis zu } 20 \text { Blumentöpfe } \\
\text { können an das Gateway } \\
\text { konnektiert werden. Das } \\
\text { Gateway kann die Daten } \\
\text { über eine Internet-Cloud, } \\
\text { Smartphones oder } \\
\text { ähnlichem zur Verfügung } \\
\text { stellen. }\end{array}$ \\
\hline
\end{tabular}

Zukünftig soll das System dahingehend erweitert werden, dass damit das Umgebungslicht und der Nährstoffgehalt des Bodens gemessen werden kann. Zudem soll eine Datenbank programmiert werden, in der für unterschiedliche Pflanzentypen genaue Randbedingungen für die Pflege der Pflanze enthalten sind. Aufgrund dieser Datenbank soll die Elektronik des Systems für jede individuelle Pflanze die optimalen Parameter für die Pflege der Pflanze einstellen (z.B. Menge der Bewässerung), oder den Anwender darüber informieren, falls ein Optimum nicht erreicht ist (z.B. zu viel Schatten).

\section{Literaturnachweis}

[1] J.R. Rosslin, K. Tai-hoon, "Applications, systems, and methods in smart home technology: a review" International Journal of Advanced Science and Technology, Vol. 15, 2010.

[2] P.-P. Ray: Internet of things for smart agriculture: Technologies, practices and future direction. In Journal of Ambient Intelligence and Smart Environments, vol. 9, no. 4, pp. 395-420, 2017; DOI: 10.3233/AIS-170440
[3] A. H. Abbas, M. M. Mohammed, G. M. Ahmed, E. A. Ahmed and R. A. A. A. Abul Seoud, "Smart watering system for gardens using wireless sensor networks," 2014 International Conference on Engineering and Technology (ICET), Cairo, 2014, pp. 1-5.; DOI: 10.1109/ICEngTechnol.2014.7016780

[4] F. Caetano, R. Pitarma and P. Reis, "Intelligent management of urban garden irrigation," 2014 9th Iberian Conference on Information Systems and Technologies (CISTI), Barcelona, 2014, pp. 1-6.; Doi: 10.1109/CISTI.2014.6876907

[5] G. Carrión, M. Huerta and B. Barzallo, "Monitoring and irrigation of an urban garden using IoT," 2018 IEEE Colombian Conference on Communications and Computing (COLCOM), Medellin, 2018, pp. 1-6.; DOI: 10.1109/ColComCon.2018.8466722

[6] V. Biswal, H. M. Singh, W. Jeberson, A. S. Dhar, "Greeves: A Smart Houseplant Watering and Monitoring System", International Journal of Science, Engineering and Technology Research (IJSETR), Volume 4, Issue 7, July 2015 ISSN: 2278 - 7798, 2015

[7] S. N. Ishak, N. N. N. A. Malik, N. M. A. Latiff, N. E. Ghazali and M. A. Baharudin, "Smart home garden irrigation system using Raspberry Pi," 2017 IEEE 13th Malaysia International Conference on Communications (MICC), Johor Bahru, 2017, pp. 101-106; DOI: 10.1109/MICC.2017.8311741.

[8] Daisy.si: http://daisy.si/dv1/Daisy_dv1_EN_rv0.2.pdf (User Manual; Last Access: 05.03.19).

[9] Parrot: https://www.parrot.com/global/connectedgarden/parrot-pot\#app (Last Access: 18.10.18).

[10] P. Moser: „Entwicklung einer drahtlosen Sensor-Netzwerk-Plattform für den LowPower-Anwendungsbereich", Bachelor Thesis.Offenburg, 2017.

[11] S. Möhringer, P. Moser, E. Mackensen, "Indoor Smart Gardening based on an Energy Autonomous Wireless Network Platform" In: Wireless Congress 2018: Systems \& Application, Conference Proceedings, ICM International Congress Center München, Munich, Germany, November 2018.

[12] E. Mackensen, M. Lai and T. M. Wendt, "Bluetooth Low Energy (BLE) based wireless sensors," SENSORS, 2012 IEEE, Taipei, 2012, pp. 1-4. DOI: 10.1109/ICSENS.2012.6411303

[13] T. Wendt, F. Volk and E. Mackensen, "A benchmark survey of long range (LoRaTM) spread-spectrum-communication at $2.45 \mathrm{GHz}$ for safety applications," 2015 IEEE 16th Annual Wireless and Microwave Technology Conference (WAMICON), Cocoa Beach, FL, 2015, pp. 1-4. DOI:

10.1109/WAMICON.2015.7120312 